\title{
Homeostatic control of immunity by TCR peptide-specific Tregs
}

\author{
Vipin Kumar
}

Laboratory of Autoimmunity, Torrey Pines Institute for Molecular Studies, San Diego, California, USA.

\begin{abstract}
Regulation of the immune response is a multifaceted process involving lymphocytes that function to maintain both self tolerance as well as homeostasis following productive immunity against microbes. There are 2 broad categories of Tregs that function in different immunological settings depending upon the context of antigen exposure and the nature of the inflammatory response. During massive inflammatory conditions such as microbial exposure in the gut or tissue transplantation, regulatory $\mathrm{CD}^{+} \mathrm{CD}^{+} 5^{+}$Tregs broadly suppress priming and/or expansion of polyclonal autoreactive responses nonspecifically. In other immune settings where initially a limited repertoire of antigen-reactive $T$ cells is activated and expanded, TCR-specific negative feedback mechanisms are able to achieve a fine homeostatic balance. Here I will describe experimental evidence for the existence of a Treg population specific for determinants that are derived from the TCR and are expressed by expanding myelin basic protein-reactive $T$ cells mediating experimental autoimmune encephalomyelitis, an animal prototype for multiple sclerosis. These mechanisms ensure induction of effective but appropriately limited responses against foreign antigens while preventing autoreactivity from inflicting escalating damage. In contrast to $\mathrm{CD}^{+} 5^{+}$Tregs, which are most efficient at suppressing priming or activation, these specific Tregs are most efficient in controlling T cells following their activation.
\end{abstract}

\section{A historical perspective on specific Tregs in feedback regulation}

Feedback inhibition in macromolecular synthetic pathways has been described as the mechanism by which the end product either inhibits formation of or suppresses enzymes in its biosynthetic pathway, thus specifically limiting the accumulation of that end product. The idea proposed by Neils Jerne (1) that specialized lymphocytes might inhibit immune responses gave rise to the description of suppressor T cells in vitro in the 1960s and 1970s (2-5). Some of the initial indications of the existence of Tregs in vivo came from studies in the mid-1970s involving alloresponses wherein vaccination with polyclonal $\mathrm{T}$ lymphoblasts from a parental strain into $F_{1}$ hybrid rats abrogated anti-alloresponse and graft rejection $(6,7)$. These findings were followed by the demonstration that vaccination with an attenuated myelin basic proteinreactive (MBP-reactive) but not a purified protein derivative of mycobacterium-reactive cloned T cell line prevented MBP-induced experimental autoimmune encephalomyelitis (EAE) in Lewis rats, further suggesting the induction of immunity against the antigen receptors on autoimmune lymphocytes (8). Cytotoxic $\mathrm{CD}^{+} \mathrm{T}$ cell lines capable of responding to $\mathrm{T}$ cells were induced in rats recovering from graft-versus-host disease or from T cell-mediated EAE ( 9 , 10). Thus, killing of encephalitogenic $\mathrm{CD}^{+} \mathrm{T}$ cell lines in vitro by cytotoxic $\mathrm{CD}^{+}$as well as neutralization of their capacity in vivo to cause EAE clearly indicated that $\mathrm{CD}^{+}$Tregs recognizing some cell surface molecules on vaccinating $\mathrm{CD}^{+}$can be induced. Moreover in human studies, $\mathrm{CD}^{+} \mathrm{T}$ cells, which were isolated from $\mathrm{CD} 4^{+} \mathrm{T}$ cell-vaccinated subjects, specifically lysed the inciting $\mathrm{CD}^{+} \mathrm{T}$ cells

Nonstandard abbreviations used: CDR, complementarity determining region; EAE, experimental autoimmune encephalomyelitis; Fr, framework; MBP, myelin basic protein.

Conflict of interest: The author has declared that no conflict of interest exists.

Citation for this article: J. Clin. Invest. 114:1222-1226 (2004).

doi:10.1172/JCI200423166. in vitro, and accordingly vaccination resulted in a decrease in the frequency of MBP-reactive T cells in peripheral blood lymphocytes (11). Consistent with the role of $\mathrm{CD}^{+}$Tregs, mice functionally deficient in $\mathrm{CD}^{+} \mathrm{T}$ cells, either by gene targeting or by antibody depletion, were shown to develop a higher frequency of relapses and lost resistance to reinduction of EAE upon secondary immunization $(12,13)$. These data suggest a negative feedback regulatory mechanism involving a regulatory $\mathrm{T}$ cell population that is induced by a pathogenic T cell itself ("suicidal induction") and may be specific for molecules on the aggressive $T$ cells. These molecules are either activation "ergotypic" markers or antigen receptors (TCRs) on $\mathrm{T}$ cells $(14,15)$. Recent data further suggest that these Tregs are also present in naive animals and can be further expanded in response to antigen-activated $\mathrm{T}$ cells (ref. 16; Madakamutil and Kumar, unpublished data).

\section{Specificity for regulation is provided by the recognition of TCR peptide/MHC complexes}

Most work on the characterization of the specific Tregs comes from studies of the regulation of the anti-MBP response mediating EAE in Lewis rats or in B10.PL or PL/J mice. There are 3 critical features in these model systems that allow examination of whether TCR peptides are part of the target structures recognized by Tregs: (a) initially, the immune response to $\mathrm{MBP}$ is primarily targeted to a single immunodominant determinant $(17,18)$; (b) pathogenic $\mathrm{T}$ cells recognizing this immunodominant peptide predominantly express the TCR $V_{\beta} 8.2$ chain (19-22); and (c) the clinical disease is largely monophasic, and most animals spontaneously recover and are resistant to reinduction of EAE. Thus, in both the Lewis rat and murine models of EAE, immunization with peptides derived from the TCR $V_{\beta} 8.2$ chain resulted in the induction of $\mathrm{CD}^{+}$and $\mathrm{CD}^{+}$Treg responses that lead to protection from EAE; this suggests that the TCR or peptides derived from the TCR of autoimmune $T$ cells can be targeted for recognition by Tregs (23-29). It is not yet known whether the mechanisms or 
the phenotype of Tregs following the T cell-vaccination protocol or following TCR-peptide vaccination are the same.

TCR peptides derived mostly from the conserved complementarity determining region (CDR) and framework ( $\mathrm{Fr}$ ) regions of the TCR $V_{\beta}$-chains have been found to be immunogenic in both animals and in humans (23-35). Although the phenotype or the MHC restriction of Tregs has not been defined in most cases, it is clear that peptides from 2 distinct conserved regions, namely the Fr3 and CDR $1 / 2$ regions on the TCR $V_{\beta} 8.2$ chain, can induce regulation and protection from antigen-induced EAE in both rats and B10.PL mice $(15,26,27,29,30,36-40)$. It is noteworthy that in humans, both the CDR2 and Fr3 regions have been found to be immunogenic and can potentially induce Treg activity $(34,35,41)$.

A better understanding of the antigen specificity and $\mathrm{MHC}$ restriction of Tregs at the clonal level comes from studies of regulation of the anti-MBP response in B10.PL mice (see Figure 1). Two distinct populations of Tregs that collaborate to control EAE have been characterized. $\mathrm{CD}^{+}$Tregs are specific for a determinant from the Fr3 region (peptide B5, AAs 76-101) that binds to the MHC class II molecule I- $\mathrm{A}^{\mathrm{u}}$. The $\mathrm{CD} 4^{+}$Tregs are predominantly $\mathrm{V}_{\beta} 14^{+}$and help in the recruitment or activation of $\mathrm{CD}^{+}$Tregs, which ultimately deplete activated MBP-reactive pathogenic $\mathrm{V}_{\beta} 8.2^{+} \mathrm{CD}^{+} \mathrm{Th} 1$ cells. $\mathrm{CD}^{+}$Tregs recognize a different determinant from the CDR1/2 region (p42-50, AAs $42-50$ of the $V_{\beta} 8.2$ chain) in the context of a nonclassical MHC class Ib molecule, Qa-1 ${ }^{a}$ (Tang and Kumar, unpublished data). Nonclassical class I molecules are less polymorphic in comparison to the classical class I molecules. $\mathrm{CD}^{+} \mathrm{T}$ cell hybridomas reactive to $\mathrm{V}_{\beta} 8.2^{+} \mathrm{T}$ cells isolated from $\mathrm{T}$ cell-vaccinated mice have been shown to be restricted by the Qa-1 MHC class Ib molecules (42-44). Evidence that Qa-1-restricted $\mathrm{CD}^{+}$Tregs are actually involved in the regulation of autoimmune disease comes from experiments in which adoptive transfer of $\mathrm{Qa}-1^{\mathrm{a}}$-restricted $\mathrm{CD} 8^{+} \mathrm{T}$ cell clones reactive to the $\mathrm{p} 42-50$ peptide from the $\mathrm{V}_{\beta} 8.2 \mathrm{TCR}$ prevents MBP-induced EAE in syngeneic recipients (Tang et al., unpublished data). These functional $\mathrm{CD}^{+}$Tregs are $\mathrm{Qa}-1^{\mathrm{a}}$ restricted despite the ability of some TCR peptides to induce class Ia-restricted $\mathrm{CD}^{+} \mathrm{T}$ cells (45). They also express CD8 $\alpha \alpha$ homodimers (Tang and Kumar, unpublished data), a characteristic of intraepithelial lymphocytes in the intestinal mucosa (46). Recognition of TCR/Qa-1 complexes on T cells raises a number of important issues: for example, have T cells developed specialized processing and loading machinery for a preferential surface display of TCR peptide/Qa-1 complexes following activation; and are activation, differentiation, or amplification of Qa-1-restricted $\mathrm{CD}^{+}$Tregs more dependent upon help provided by $\mathrm{CD}^{+}$Tregs than class Ia-restricted $\mathrm{CD}^{+} \mathrm{T}$ cells?

Two sets of experiments clearly suggested that TCR peptide-reactive Tregs are naturally involved in the negative feedback regulation of the anti-MBP response and mediate recovery from EAE: (a) Tregs expand naturally during the course of EAE, and recovery from disease can be rapidly accelerated by immunization with either the Fr3 or CDR1/2 TCR peptide in both rats and mice $(26-28,38,47)$; and (b) since $\mathrm{CD}^{+}$Tregs expressed limited TCR $V_{\beta}$ gene segments, antibodies against these $V_{\beta}$ chains were used to specifically deplete regulatory $T$ cells. Depletion of Tregs resulted in poor recovery and increased severity of relapsing paralysis $(28,39)$. These studies were complemented by experiments demonstrating that the adoptive transfer of $\mathrm{CD}^{+}{ }^{+}$Treg clones into WT but not into $\mathrm{CD}^{+} \mathrm{T}$ cell-knockout mice result in significant protection from antigen-induced EAE (26).

\section{Specific regulation is mediated by depletion of activated MBP-reactive CD4+ lymphocytes by CD8+ Tregs}

Self antigen-reactive $\mathrm{T}$ cells can be downregulated by several mechanisms, including the induction of anergy, which is a loss of the ability to respond to a particular antigen; and cellular depletion or deviation toward a type 2 cytokine secretion phenotype (48-50). Although in vivo studies using TCR-peptide vaccination suggested induction of anergy $(51)$ in the target $\mathrm{V}_{\beta} 8.2^{+} \mathrm{T}$ cell population, $\mathrm{CD}^{+}$Treg lines and hybridomas generated following $\mathrm{T}$ cell vaccination show in vitro killing of these cells $(10,42)$. Recent studies examining the fate of MBP-reactive $V_{\beta} 8.2^{+} \mathrm{T}$ cells using CFSE labeling as well as immunoscope analysis clearly demonstrate that the target $\mathrm{V}_{\beta} 8.2^{+} \mathrm{T}$ cells undergo apoptotic depletion in vivo following expansion of $\mathrm{CD}^{+}$and $\mathrm{CD}^{+}$Tregs in B10.PL mice (16) (Figure 1). These in vivo findings are consistent with in vitro data showing specific killing of $\mathrm{V}_{\beta} 8.2^{+}$but not $\mathrm{V}_{\beta} 8.2^{-} \mathrm{T}$ cell targets by TCR peptide-reactive $\mathrm{CD}^{+} \mathrm{T}$ cell clones (Tang and Kumar, unpublished data) as well as by $\mathrm{CD}^{+}$hybridomas generated from

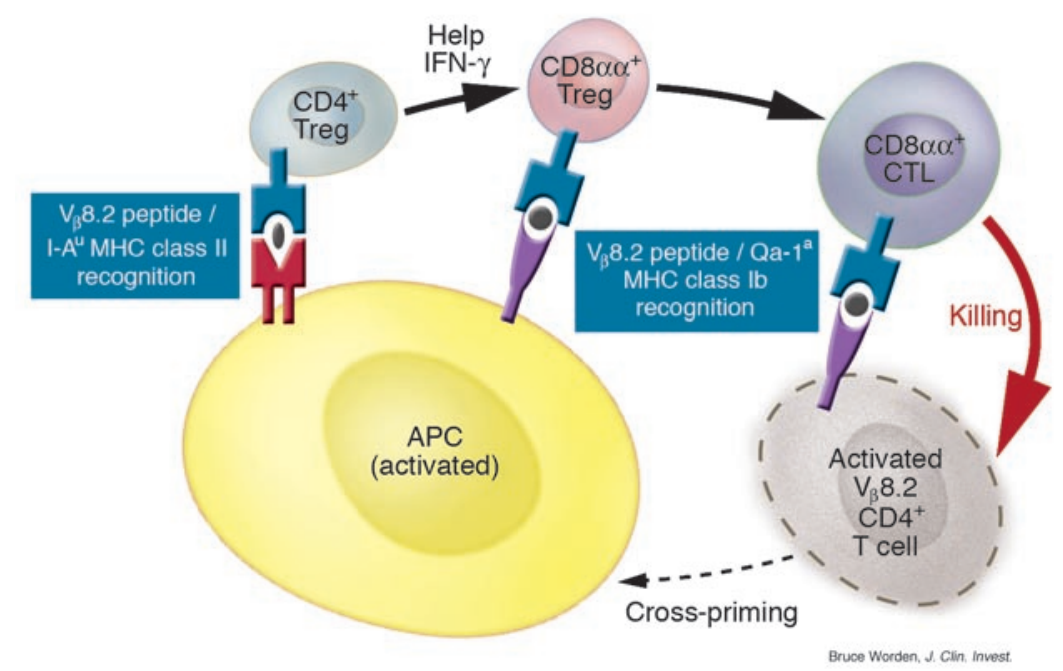

Figure 1

A negative-feedback regulatory mechanism involving CD4+ and CD8+ Tregs recognizing TCR peptide/MHC complexes. During normal peripheral turnover or following the expansion/contraction phase, MBP-reactive, $\mathrm{V}_{\beta} 8.2^{+} \mathrm{CD} 4^{+} \mathrm{T}$ cells are captured by professional APCs. These APCs process and present distinct TCR $\mathrm{V}_{\beta} 8.2$ peptides in the context of I-A ${ }^{u} \mathrm{MHC}$ class II and Qa-1 ${ }^{a} \mathrm{MHC}$ class Ib molecules for the induction of $\mathrm{CD}^{+}$and $\mathrm{CD}^{+}$Tregs, respectively, a process commonly referred to as cross-priming. CD4 $4^{+}$Tregs predominantly utilize the TCR $\mathrm{V}_{\beta} 14$ gene segment, recognize an Fr3 region TCR peptide, and secrete type 1 proinflammatory cytokines, such as IFN- $\gamma$, for effective recruitment or activation of $\mathrm{CD}^{+}$Tregs. CD8+ Tregs recognize CDR1/2 region TCR peptide/Qa- $1^{\text {a }}$ complexes on the surface of activated and pathogenic $\mathrm{V}_{\beta} 8.2^{+}$Th1 cells, resulting in their apoptotic death. Low avidity, slower-reacting Th2 cells that are relatively less susceptible to apoptosis can then eventually expand, resulting in immune deviation of the anti-MBP response at the population level. At this stage, Th2 cell secretion of cytokines such as IL-4 or IL-10 can further enhance the downregulation of the anti-MBP response (41). 
$\mathrm{T}$ cell-vaccinated mice (42). Significantly, only activated $\mathrm{V}_{\beta} 8.2^{+} \mathrm{T}$ cells, but not naive $\mathrm{V}_{\beta} 8.2^{+}$or activated $\mathrm{V}_{\beta} 13^{+} \mathrm{T}$ cells, are depleted following regulation (16). Consistent with the requirement for $\mathrm{CD}^{+}$Tregs in depletion of the dominant pathogenic $\mathrm{V}_{\beta} 8.2^{+}$ $\mathrm{T}$ cells, expansion of MBP-reactive $\mathrm{V}_{\beta} 8.2^{+} \mathrm{T}$ cell clones occurs in $\mathrm{CD}^{+} \mathrm{T}$ cell-depleted animals (52). Therefore, the regulation mediated by Tregs does not influence the majority of the $\mathrm{V}_{\beta} 8.2^{+} \mathrm{T}$ cells (which are nonactivated) but only those that are activated by MBP. It is significant that CD $8^{+}$Tregs appear to modulate only Th 1 cells and not Th2 cells both in vitro as well as in vivo $(53,54)$. This does not appear to be due to a difference in the TCR or Qa-1 expression but may involve either differential TCR processing or different recognition motifs. Furthermore, Th1 cells are considerably more sensitive to apoptosis than Th2 cells.

$\mathrm{CD}^{+}$Tregs provide crucial help for the recruitment of $\mathrm{CD}^{+}$ Tregs, which are the ultimate effectors of regulation (Figure 1). Interestingly, $\mathrm{Fr} 3$ peptide-reactive $\mathrm{CD}^{+}{ }^{+}$Treg clones secrete large amounts of IL- 2 and IFN- $\gamma$, but barely detectable levels of IL-4 and IL-5, and do not secrete IL-10 or TGF- $\beta(26,54)$. Accordingly, a $\mathrm{CD}^{+}$Treg response of the type 1 sort is required in vivo for effective regulation and prevention of disease. If these $\mathrm{CD} 4^{+}$Tregs are forced to deviate in a type 2 direction, mice contract exacerbated EAE, and most die from paralysis without recovery $(54,55)$. Recent experiments using $\mathrm{CD}^{+}$Tregs from IFN- $\gamma$-knockout mice further suggest that secretion of IFN- $\gamma$ by $\mathrm{CD}^{+}$Tregs is absolutely required for this regulation (Pedersen and Kumar, unpublished data).

The depletion of high-avidity activated Th1 cells by CD8 ${ }^{+}$Tregs enables a relatively slower reacting compartment of low-avidity, MBP-reactive Th2 cells (which may or may not express $V_{\beta} 8.2$ ) to expand, resulting in immune deviation of the anti-MBP response $(40,54,56,57)$. Thus, the eventual outcome of TCR-based regulation is the deviation of MBP-reactive T cells at the bulk population level in a Th2 direction. This may explain why TCR-based regulation directed to a single $V_{\beta}$ chain is able to control diseaseinducing $\mathrm{T}$ cells that use other TCR $\mathrm{V}_{\beta}$ chains, thereby providing a suppressive environment for responses to other determinants from the same protein as well as from other myelin components that may arise as a result of determinant spreading during chronic demyelination (58). Consistent with this, Th2 deviation of antigenspecific $\mathrm{T}$ cells using an altered peptide ligand has been shown to result in the disappearance of the bulk of the $\mathrm{T}$ cell infiltrate from the CNS and reversal of EAE (59). Therefore targeting of only a few dominant autoreactive $T$ cells can abort autoimmune pathology.

\section{TCR peptide/MHC complex recognition is crucial for the induction and function of Tregs}

How are TCR-reactive Tregs naturally primed in vivo? In light of recent studies (ref. 44; Tang and Kumar, unpublished data), it is reasonable to predict that the cell-surface display of TCR peptide/Qa-1 complexes on the activated target $\mathrm{CD}^{+} \mathrm{T}$ cells is required for their specific recognition and eventual killing by $\mathrm{CD}^{+}$Tregs (Figure 1). Although it has been suggested that Qa- $1^{+}$-activated T cells themselves can prime $\mathrm{CD}^{+}$Tregs directly (44), there is little in vivo evidence demonstrating priming of naive $\mathrm{CD}^{+}$or $\mathrm{CD}^{+} \mathrm{T}$ cells by lymphocytes in the absence of professional APCs. Therefore, I would like to propose that presentation of TCR peptides by professional APCs such as dendritic cells, macrophages, or B cells, is essential for the cross-priming of both $\mathrm{CD}^{+}$and $\mathrm{CD} 8^{+}$Treg. The same APC could present TCR determinants in MHC class Ib and class II contexts to effectively prime both $\mathrm{CD}^{+}$Tregs and $\mathrm{CD} 4^{+}$Tregs, respectively. A critical aspect in TCR-centered regulation might be the presence of suitable processing sites in the TCR $V_{\beta}$ framework or in the CDR1/2 regions. Examination of these determinants in the Fr3 and CDR2 regions of the $V_{\beta} 8.2$ chain suggests that they may be particularly available for processing, since they possess endopeptidase target residues that would permit their cleavage.

How general is the mechanism of feedback regulation based on the recognition of TCR peptide/MHC complexes? Can peptides derived from other TCR $V_{\beta}$ chains be processed and presented in sufficient quantity to generate specific Tregs? Is display of TCR peptides on APCs limited to particular $V_{\beta}$ families with a high frequency in the periphery? Since $\mathrm{V}_{\beta} 8$-expressing $\mathrm{T}$ cells in most mice constitute almost $20-30 \%$ of the total peripheral $\mathrm{T}$ cell repertoire, it is possible that professional APCs can capture apoptotic T cells during normal peripheral turnover and process and present TCR determinants in both MHC class Ib and class II contexts. A similar situation occurs during antiviral immune response, where viral determinant-reactive $T$ cells using certain TCR $V_{\beta}$ chains expand rapidly and can occupy up to $50 \%$ of the peripheral repertoire (60). Antigen-induced cell death of the large number of these activated $\mathrm{T}$ cells may result in cross-priming of anti-TCR CD $4^{+}$and $\mathrm{CD}^{+} \mathrm{T}$ cell responses. It is possible that the depletion of activated viral antigen-reactive $T$ cells in the contraction phase of the response that is followed by a successful anti-viral response may be augmented by TCR peptide-reactive $\mathrm{CD}^{+}$Tregs. Therefore, this regulation may be a generalized mechanism involved in the establishment of homeostasis following infections, transplantation, and an autoimmune response.

\section{Use of TCR delivery systems can be exploited for specific intervention in T cell-mediated immune pathologies}

Priming of TCR peptide-reactive Tregs following vaccination with disease-related T cells or their TCRs has been demonstrated both to prevent and to ameliorate autoimmune disease in experimental animals, which has led to clinical trials of TCR-based vaccinations in MS and RA in humans (41, 61-63). Clinical trials of T cell vaccination in MS patients are currently ongoing (L. Weiner, University of Southern California, Los Angeles, California, USA, and J. Zhang, Baylor College of Medicine, Houston, Texas, USA; personal communication). Success of these trials will depend upon the identification of dominant pathogenic T cell clones as well as relevant TCR peptides for use in vaccination. Although class Ib molecules, for example, Qa-1 in mouse and its equivalent HLA-E in humans, are highly conserved, it is likely that in different haplotypes, different TCR peptides might be targeted by the MHC class II-restricted CD4 ${ }^{+}$Treg population. Therefore, therapeutic delivery of an entire TCR $V_{\beta}$ chain may be more broadly effective and less cumbersome than the individual peptides. This is especially true for those situations where response to an antigen consists of $T$ cells that utilize several predominant TCR $V_{\beta}$ regions. In fact, delivery of multiple TCR $V_{\beta}$ chains has been shown to be more effective than a single $V_{\beta}$ chain in modulating autoimmune myocarditis mediated by a polyclonal $\mathrm{T}$ cell response (64). We and others have used different approaches in trying to determine the most efficient means of presentation of the TCR for modulation of autoimmune responses, including TCR peptides, recombinant single-chain $V_{\beta} 8.2$ proteins, $V_{\beta} 8.2$ plasmid $D N A$, and a $V_{\beta} 8.2$ adenovirus or vaccinia delivery system $(38,55-57,65,66)$.

One can predict that future investigations will reveal a detailed description of this specific regulatory process. Therefore in contrast to the generalized suppression mediated by $\mathrm{CD} 25^{+} \mathrm{CD} 4^{+}$ 
Tregs, a detailed knowledge of TCR-based regulation as well as the identification of pathogenic lymphocytes in different clinical settings will enable a search for sophisticated ways of modulating immune responses in a more targeted fashion. This will involve the engagement of naturally occurring pathways inherent in the immune system at appropriate times to obtain a desired control of the immune response. If display of the TCR peptide/MHC complex can be appropriately manipulated, TCR-based regulation should be able to be exploited for modulating a wide variety of immune responses, including those involved in transplantation, infection, tumors, and autoimmune diseases.

1. Jerne, N.K. 1984. Idiotypic networks and other preconceived ideas. Immunol. Rev. 79:5-24.

2. Gershon, R.K., and Kondo, K. 1970. Cell interactions in the induction of tolerance: the role of thymic lymphocytes. Immunology. 18:723-737.

3. Cantor, H., and Boyse, E.A. 1975. Functional subclasses of T lymphocytes bearing different Ly antigens. II. Cooperation between subclasses of $\mathrm{Ly}^{+}$ cells in the generation of killer activity. J. Exp. Med. 141:1390-1399.

4. Cantor, H., and Gershon, R.K. 1979. Immunological circuits: cellular composition. Fed. Proc. 38:2058-2064.

5. Eardley, D.D., and Gershon, R.K. 1975. Feedback induction of suppressor T-cell activity. J. Exp. Med. 142:524-529.

6. Binz, H., and Wigzell, H. 1976. Specific transplantation tolerance induced by autoimmunization against the individual's own, naturally occurring idiotypic, antigen-binding receptors. J. Exp. Med. 144:1438-1457.

7. Bellgrau, D., and Wilson, D.B. 1978. Immunological studies of T-cell receptors. I. Specifically induced resistance to graft-versus-host disease in rats mediated by host T-cell immunity to alloreactive parental T cells. J. Exp. Med. 148:103-114.

8. Ben-Nun, A., Wekerle, H., and Cohen, I.R. 1981. Vaccination against autoimmune encephalomyelitis with T-lymphocyte line cells reactive against myelin basic protein. Nature. 292:60-61.

9. Kimura, H., and Wilson, D.B. 1984. Anti-idiotypic cytotoxic T cells in rats with graft-versus-host disease. Nature. 308:463-464.

10. Sun, D., Qin, Y., Chluba, J., Epplen, J.T., and Wekerle, H. 1988. Suppression of experimentally induced autoimmune encephalomyelitis by cytolytic T-T cell interactions. Nature. 332:843-845.

11. Zhang, J., Medaer, R., Stinissen, P., Hafler, D., and Raus, J. 1993. MHC-restricted depletion of human myelin basic protein-reactive $\mathrm{T}$ cells by $\mathrm{T}$ cell vaccination. Science. 261:1451-1454.

12. Koh, D.-R., et al. 1992. Less mortality but more relapses in experimental allergic encephalomyeltis in CD8-/- mice. Science. 256:1210-1213.

13. Jiang, H., Zhang, S.I., and Pernis, B. 1992. Role of CD8+ T cells in murine experimental allergic encephalomyelitis. Science. 256:1213-1215.

14. Lohse, A.W., et al. 1993. Induction of the antiergotypic response. Int. Immunol. 5:533-539.

15. Kumar, V., and Sercarz, E. 1993. T cell regulatory circuitry: antigen-specific and TCR-idiopeptide- specific $\mathrm{T}$ cell interactions in EAE. Int. Rev. Immunol. 9:287-297.

16. Madakamutil, L.T., Maricic, I., Sercarz, E., and Kumar, V. 2003. Regulatory T cells control autoimmunity in vivo by inducing apoptotic depletion of activated pathogenic lymphocytes. J. Immunol. 170:2985-2992.

17. Kibler, R.F., et al. 1977. Immune response of Lewis rats to peptide $\mathrm{C} 1$ (residues 68-88) of guinea pig and rat myelin basic proteins. J. Exp. Med. 146:1323-1331.

18. Zamvil, S.S., et al. 1986. T-cell epitope of the autoantigen myelin basic protein that induces encephalomyelitis. Nature. 324:258-260.

19. Urban, J.L., et al. 1988. Restricted use of T cell receptor $V$ genes in murine autoimmune encephalomyelitis raises possibilities for antibody therapy. Cell. 54:577-592.

20. Acha-Orbea, H., et al. 1988. Limited heterogeneity of $\mathrm{T}$ cell receptors from lymphocytes mediating autoimmune encephalomyelitis allows specific immune intervention. Cell. 54:263-273.

21. Kumar, V., Kono, D.H., Urban, J.L., and Hood, L. 1989. The T-cell receptor repertoire and autoimmune diseases. Annu. Rev. Immunol. 7:657-682.

22. Burns, F.R., et al. 1989. Both rat and mouse T cell receptors specific for the encephalitogenic determinant of myelin basic protein use similar $\mathrm{V}$ and $\mathrm{V}$ chain genes. J. Exp. Med. 169:27-39.

23. Howell, M.D., et al. 1989. Vaccination against experimental allergic encephalomyelitis with $\mathrm{T}$ cell receptor peptides. Science. 246:668-670.

24. Vandenbark, A.A., Hashim, G., and Offner, H. 1989. Immunization with a synthetic T-cell receptor Vregion peptide protects against experimental autoimmune encephalomyelitis. Nature. 341:541-544.

25. Karpus, W.J., Gould, K.E., and Swanborg, R.H. 1992. CD4+ suppressor cells of autoimmune encephalomyelitis respond to $\mathrm{T}$ cell receptor-associated determinants on effector cells by interleukin4 secretion. Eur. J. Immunol. 22:1757-1763.

26. Kumar, V., and Sercarz, E. 1993. The involvement of TCR-peptide-specific regulator $\mathrm{CD}^{+} \mathrm{T}$ cells in recovery from antigen-induced autoimmune disease. J. Exp. Med. 178:909-916.

27. Kumar, V., Tabibiazar, R., Geysen, H.M., and Sercarz, E. 1995. Immunodominant framework region 3 peptide from TCR $V$ beta 8.2 chain controls murine experimental autoimmune encephalomyelitis. J. Immunol. 154:1941-1950.

28. Kumar, V., Stellrecht, K., and Sercarz, E. 1996. Inactivation of T cell receptor peptide-specific CD4 regulatory $\mathrm{T}$ cells induces chronic experimental autoimmune encephalomyelitis (EAE). J. Exp. Med. 184:1609-1617.

29. Kumar, V., Aziz, F., Sercarz, E., and Miller, A. 1997. Regulatory $\mathrm{T}$ cells specific for the same framework 3 region of the Vbeta8.2 chain are involved in the control of collagen II-induced arthritis and experimental autoimmune encephalomyelitis. J. Exp. Med. 185:1725-1733.

30. Vainiene, M., et al. 1996. Neonatal injection of Lewis rats with recombinant $\mathrm{V}$ beta 8.2 induces $\mathrm{T}$ cell but not B cell tolerance and increased severity of experimental autoimmune encephalomyelitis. J. Neurosci. Res. 45:475-486.

31. MacNeil, D., Fraga, E., and Singh, B. 1993. Characterization of murine $T$ cell responses to peptides of the variable region of self $\mathrm{T}$ cell receptor betachains. J. Immunol. 151:4045-4054.

32. Jesson, M.I., et al. 1998. The immune response to soluble D10 TCR: analysis of antibody and T cell responses. Int. Immunol. 10:27-35.

33. Falcioni, F., et al. 1995. Self tolerance to T cell receptor V beta sequences. J. Exp. Med. 182:249-254.
34. Saruhan-Direskeneli, G., et al. 1993. Human T cell autoimmunity against myelin basic protein: $\mathrm{CD} 4+$ cells recognizing epitopes of the $\mathrm{T}$ cell receptor beta chain from a myelin basic protein-specific $\mathrm{T}$ cell clone. Eur. J. Immunol. 23:530-536.

35. Zang, Y.C., Hong, J., Rivera, V.M., Killian, J., and Zhang, J.Z. 2000. Preferential recognition of TCR hypervariable regions by human anti-idiotypic $\mathrm{T}$ cells induced by $\mathrm{T}$ cell vaccination. J. Immunol. 164:4011-4017.

36. Vandenbark, A.A., Hashim, G.A., and Offner, H. 1996. T cell receptor peptides in treatment of autoimmune disease: rationale and potential. J. Neurosci. Res. 43:391-402.

37. Kumar, V., and Sercarz, E. 1996. Dysregulation of potentially pathogenic self reactivity is crucial for the manifestation of clinical autoimmunity. J. Neurosci. Res. 45:334-339.

38. Kumar, V., et al. 1997. Recombinant T cell receptor molecules can prevent and reverse experimental autoimmune encephalomyelitis: dose effects and involvement of both CD4 and CD8 T cells. J. Immunol. 159:5150-5156.

39. Kumar, V. 1998. TCR peptide-reactive T cells and peripheral tolerance to myelin basic protein. Res. Immunol. 149:827-834; discussion 852-854, $855-860$.

40. Kumar, V., and Sercarz, E. 2001. An integrative model of regulation centered on recognition of TCR peptide/MHC complexes. Immunol. Rev. 182:113-121.

41. Vandenbark, A.A., et al. 1996. Treatment of multiple sclerosis with T-cell receptor peptides: results of a double-blind pilot trial. Nat. Med. 2:1109-1115.

42. Jiang, H., et al. 1995. Murine CD8+ T cells that specifically delete autologous CD4+ T cells expressing $\mathrm{V}$ beta 8 TCR: a role of the Qa-1 molecule. Immunity. 2:185-194.

43. Jiang, H., et al. 1998. T cell vaccination induces $T$ cell receptor Vbeta-specific Qa-1- restricted regulatory CD8(+) T cells. Proc. Natl. Acad. Sci. U. S. A. 95:4533-4537.

44. Jiang, H., and Chess, L. 2000. The specific regulation of immune responses by CD8+ T cells restricted by the MHC class Ib molecule, Qa-1. Annu. Rev. Immunol. 18:185-216.

45. Kuhrober, A., Schirmbeck, R., and Reimann, J. 1994. Vaccination with T cell receptor peptides primes anti-receptor cytotoxic $\mathrm{T}$ lymphocytes (CTL) and anergizes $T$ cells specifically recognized by these CTL. Eur. J. Immunol. 24:1172-1180.

46. Gapin, L., Cheroutre, H., and Kronenberg, M. 1999. Cutting edge: TCR alpha beta+ CD8 alpha alpha+ $\mathrm{T}$ cells are found in intestinal intraepithelial lymphocytes of mice that lack classical MHC class I molecules. J. Immunol. 163:4100-4104.

47. Offner, H., Hashim, G.A., and Vandenbark, A.A 1991. T cell receptor peptide therapy triggers autoregulation of experimental encephalomyelitis. Science. 251:430-432.

48. Mueller, D.L., Jenkins, M.K., and Schwartz, R.H. 1989. Clonal expansion versus functional clonal inactivation: a costimulatory signalling pathway 
determines the outcome of $\mathrm{T}$ cell antigen receptor occupancy. Annu. Rev. Immunol. 7:445-480.

49. Kappler, J.W., Roehm, N., and Marrack, P. 1987. T cell tolerance by clonal elimination in the thymus. Cell. 49:273-280.

50. Coffman, R.L., Varkila, K., Scott, P., and Chatelain, R. 1991. Role of cytokines in the differentiation of CD4+ T-cell subsets in vivo. Immunol. Rev. 123:189-207.

51. Gaur, A., Ruberti, G., Haspel, R., Mayer, J.P., and Fathman, C.G. 1993. Requirement for CD8+ cells in $\mathrm{T}$ cell receptor peptide-induced clonal unresponsiveness. Science. 259:91-94.

52. Jiang, H., et al. 2003. Regulatory CD8+ T cells finetune the myelin basic protein-reactive $\mathrm{T}$ cell receptor $\mathrm{V}$ beta repertoire during experimental autoimmune encephalomyelitis. Proc. Natl. Acad. Sci.U. S. A. 100:8378-8383.

53. Jiang, H., Braunstein, N.S., Yu, B., Winchester, R., and Chess, L. 2001. CD8+ T cells control the TH phenotype of MBP-reactive CD4+ T cells in EAE mice. Proc. Natl. Acad. Sci. U. S. A. 98:6301-6306.

54. Kumar, V., and Sercarz, E. 1998. Induction or protection from experimental autoimmune encephalomyelitis depends on the cytokine secretion profile of TCR peptide-specific regulatory CD4 T cells. J. Immunol. 161:6585-6591.
55. Braciak, T.A., et al. 2003. Protection against experimental autoimmune encephalomyelitis generated by a recombinant adenovirus vector expressing the $\mathrm{V}$ beta $8.2 \mathrm{TCR}$ is disrupted by coadministration with vectors expressing either IL- 4 or -10 . J. Immunol. 170:765-774.

56. Waisman, A., et al. 1996. Suppressive vaccination with DNA encoding a variable region gene of the $\mathrm{T}$-cell receptor prevents autoimmune encephalomyelitis and activates Th2 immunity. Nat. Med. 2:899-905

57. Kumar, V., et al. 2001. Induction of a type 1 regulatory CD4 $\mathrm{T}$ cell response following $\mathrm{V}$ (beta) 8.2 DNA vaccination results in immune deviation and protection from experimental autoimmune encephalomyelitis. Int. Immunol. 13:835-841.

58. Lehmann, P.V., Forsthuber, T., Miller, A., and Sercarz, E.E. 1992. Spreading of T-cell autoimmunity to cryptic determinants of an autoantigen. Nature. 358:155-157.

59. Brocke, S., et al. 1996. Treatment of experimental encephalomyelitis with a peptide analogue of myelin basic protein. Nature. 379:343-346.

60. Murali-Krishna, K., et al. 1998. Counting antigenspecific CD8 T cells: a reevaluation of bystander activation during viral infection. Immunity.
8:177-187.

61. Gold, D.P., et al. 1997. Results of a phase I clinical trial of a T-cell receptor vaccine in patients with multiple sclerosis. II. Comparative analysis of TCR utilization in CSF T-cell populations before and after vaccination with a TCRV beta 6 CDR2 peptide. J. Neuroimmunol. 76:29-38.

62. Zhang, I., and Raus, J. 1996. T cell vaccination in multiple sclerosis. Mult. Scler. 1:353-356.

63. Kumar, V., Sercarz, E., Zhang, J., and Cohen, I. 2001. T-cell vaccination: from basics to the clinic. Trends Immunol. 22:539-540.

64. Matsumoto, Y., Jee, Y., and Sugisaki, M. 2000. Successful TCR-based immunotherapy for autoimmune myocarditis with DNA vaccines after rapid identification of pathogenic TCR. J. Immunol. 164:2248-2254

65. Kumar, V., and Sercarz, E. 1996. Genetic vaccination: the advantages of going naked. Nat. Med. 2:857-859.

66. Chunduru, S.K., Sutherland, R.M., Stewart, G.A., Doms, R.W., and Paterson, Y. 1996. Exploitation of the Vbeta8.2 $\mathrm{T}$ cell receptor in protection against experimental autoimmune encephalomyelitis using a live vaccinia virus vector. J. Immunol. 156:4940-4945. 